\title{
PARTICLE DYNAMICS IN LOW-ENERGY TRAVELLING-WAVE LINACS
}

\author{
J.M.Corstens, A.F.J.Hammen, J.I.M. Botman \\ Eindhoven University of Technology, Cyclotron Laboratory, \\ P.O.Box 513, 5600 MB Eindhoven,The Netherlands.
}

\section{Abstract}

In this paper, we present the particle dynamics in lowenergy travelling-wave linear accelerators, applying analytical theory, based on Hamiltonian mechanics, and numerical simulations, performed by commercially available codes. The paper is an extension on earlier work, presented at EPAC'98. Cylindrical co-ordinates are used and solenoid magnetic fields are incorporated. The Hamiltonian equations of motion are given and examples of calculations are presented and compared to numerical simulations, yielding excellent agreement between both approaches.

\section{INTRODUCTION}

At the Eindhoven University of Technology, a Hamiltonian theory of particle motion of accelerated electrons in standing-wave RF structures has been developed[1]. However, this theory was only valid in principle under the assumption that the particle velocity equals the phase velocity of the main accelerating wave. This condition is not fulfilled in low-energy linear accelerators. The present, adapted Hamiltonian theory, in which also solenoidal magnetic fields are incorporated does not have this restriction. The set of equations obtained with this Hamiltonian theory is suitable for particle motion calculation. Calculations using this set of equations are compared to the results of a commercially available particle tracking code, yielding excellent agreement.

\section{THE EQUATIONS OF MOTION}

The vector potential in cilindrical co-ordinates representing electromagnetic waves in the periodic part of the linac is given by [2]:

$$
\begin{aligned}
& A_{r}=\sum_{n} \frac{k_{n}}{\omega} \frac{a_{n} E_{z}(z)}{\alpha_{n}} I_{1}\left(\alpha_{n} r\right) \cos \left(k_{n} z-\omega t\right), \\
& A_{\theta}=\sum_{j=1}^{8} A_{\theta, j}, \\
& A_{z}=-\sum_{n} \frac{a_{n} E_{z}(z)}{\omega} I_{0}\left(\alpha_{n} r\right) \sin \left(k_{n} z-\omega t\right),
\end{aligned}
$$

in which $\sum_{n}$ stands for $\sum_{n=-\infty}^{\infty}, a_{n}$ for the Fourier coefficients, $k_{n}=k_{f}+2 \pi n / d$ with $d$ the cell length and $k_{f}$ the phase shift per cell, $\alpha_{n}^{2}=k_{n}^{2}-k^{2}, E_{z}(z)$ the amplitude of the electric field in the $z$-direction. Note that this amplitude is a function of the longitudinal co-ordinate $z \cdot A_{\theta_{j}}$ is the vector potential of the static magnetic field $j$. This vector potential of solenoid $j$, expanded up to third order in $r$, reads:

$A_{\theta, j}(r, z)=\frac{1}{2} r B_{z}(r=0, z)-\frac{1}{16} r^{3} \frac{\partial^{2}}{\partial z^{2}}\left(B_{z}(r=0, z)\right)$,

with: $B_{z}(r=0, z)=-\mu_{0} \frac{\partial}{\partial z} V_{m}(z)$,

in which:

$V_{m}(z)=-\frac{N I}{2 L}\left(\sqrt{\left(z+\frac{L}{2}\right)^{2}+a^{2}}-\sqrt{\left(z-\frac{L}{2}\right)^{2}+a^{2}}\right)$,

with $\mu_{0}$ the magnetic permeability in vacuum, $I$ the current through the solenoid, $N$ the number of turns, $L$ the length and $a$ the radius of the solenoid. $z$ is defined at the axis with respect to the center of the solenoid.

Relativistic motion is described by the Hamiltonian:

$$
\begin{gathered}
H=\left\{E_{r}^{2}+\left(p_{r}-e A_{r}\right)^{2} c^{2}+\left(\frac{p_{\theta}}{r}-e A_{\theta}\right)^{2} c^{2}+\right. \\
\left.\left(p_{z}-e A_{z}\right)^{2} c^{2}\right\}^{1 / 2},
\end{gathered}
$$

where $c$ is the speed of light, $E_{r}$ the particle rest energy, $p_{r}, p_{\theta}, p_{z}$ the canonic momenta in cylindrical co-ordinates and $A_{r}, A_{\theta}, A_{z}$ the components in cylindrical co-ordinates of the vectorpotential, given by eq. 1. The new Hamiltonian becomes $K_{I}=-p_{z},-H$ and $t$ form a new pair of conjugated canonical variables. On this Hamiltonian $K_{I}$ the following scaling transformations is performed:

$$
\begin{aligned}
& \pi_{r}=\frac{c}{H_{i}} p_{r}, \pi_{\theta}=\frac{c}{H_{i}} p_{\theta}, \pi_{z}=\frac{c}{H_{i}} p_{z}, h=-\frac{H}{H_{i}}, \\
& e_{r}=\frac{E_{r}}{H_{i}}, \varepsilon_{n}=\frac{e a_{n} E_{z}(z)}{H_{i} k}, \varsigma=c t .
\end{aligned}
$$

in which $H_{i}$ is the initial energy, yielding Hamiltonian $K_{2}$ $=-\pi$ :

$$
K_{2}=-\left\{h^{2}-e_{r}^{2}-\left(\pi_{r}-e A_{r}^{*}\right)^{2}-\left(\frac{\pi_{\theta}}{r}-e A_{\theta}^{*}\right)^{2}\right\}^{1 / 2}-e A_{z}^{*},
$$

in which: $\vec{A}^{*}=\frac{c}{H_{i}} \vec{A}$.

In eq. $5 \zeta$ is transformed to $\zeta_{2}=\zeta-k_{f} z k$ (new variables are indicated by higher numerical subscripts), by generating function $G_{0}=-h \zeta_{2}-h k_{z} z k\left(h_{2}=h\right)$. Further the signs of $h_{2}$ and $\zeta_{2}$ are changed in $h_{3}$ and $\zeta_{3}$ by generating function 
$G_{1}=h_{2} \zeta_{3}$. Now $h_{3}$ is a positive quantity and $k \zeta_{3}$ has the meaning of phase. The final Hamiltonian $K_{f}$ becomes (in new co-ordinates, numerical subscripts are omitted):

$$
\begin{gathered}
K_{f}=K_{2}+\frac{\partial G_{0}}{\partial z}+\frac{\partial G_{1}}{\partial z}=\frac{k_{f}}{k} h-\left\{h^{2}-e_{r}^{2}-\right. \\
\left.\left(\pi_{r}-e A_{r}^{*}\right)^{2}-\left(\frac{\pi_{\theta}}{r}-e A_{\theta}^{*}\right)^{2}\right\}^{1 / 2}-e A_{z}^{*},
\end{gathered}
$$

in which the arguments of the components of the vector potential $\left(k_{n} z-\omega t\right)$ in eq. 1 has been replaced by $(2 \pi n z / d+k \zeta)$. The equations of scaled energy and phase are:

$$
\begin{aligned}
\frac{d h}{d z}= & -\frac{\partial K_{f}}{\partial \varsigma}=\frac{\pi_{r, k i n}}{\pi_{z, k i n}}\left(-\sum_{n} \frac{\varepsilon_{n}}{\alpha_{n}} k_{n} k I_{1}\left(\alpha_{n} r\right) \sin \left(\frac{2 \pi n}{d} z+k \varsigma\right)\right)- \\
& \sum_{n} \varepsilon_{n} k I_{0}\left(\alpha_{n} r\right) \cos \left(\frac{2 \pi n}{d} z+k \varsigma\right) \\
\frac{d \zeta}{d z}= & \frac{\partial K_{f}}{\partial h}=-\frac{h}{\pi_{z, k i n}}+\frac{k_{f}}{k}
\end{aligned}
$$

in which:

$$
\pi_{z, k i n}=\sqrt{h^{2}-e_{r}^{2}-\pi_{r, k i n}^{2}-\left(\frac{\pi_{\theta, k i n}}{r}\right)^{2}} .
$$

The transversal equation of motion for the canonical momentum reads:

$$
\begin{gathered}
\frac{d \pi_{r, c a n}}{d z}=-\frac{\partial K_{1}}{\partial r}=\frac{\pi_{r, k i n}}{\pi_{z, k i n}} e \frac{\partial A_{r}^{*}}{\partial r}+e \frac{\partial A_{z}^{*}}{\partial r}+\frac{\pi_{\theta, k i n}^{2}}{r^{3} \pi_{z, k i n}}+ \\
e \frac{\pi_{\theta, k i n}}{r^{2} \pi_{z, k i n}} \frac{\partial\left(r A_{\theta}^{*}\right)}{\partial r}
\end{gathered}
$$

This expression is inconvenient for practical use. Furthermore, $\pi_{r, c a n}$ is not of interest but $\pi_{r, k i n}$, therefore the differential equation for $\pi_{r, k i n}$ will be derived in the appendix and is given by:

$$
\begin{aligned}
\frac{d \pi_{r, k i n}}{d z}= & \sum_{n} \varepsilon_{n} \frac{I_{1}\left(\alpha_{n} r\right)}{\alpha_{n}} k\left(k-k_{n} \frac{h}{\pi_{z, k i n}}\right) \sin \left(\frac{2 \pi n}{d} z+k \zeta\right)+ \\
& \frac{\pi_{\theta, k i n}^{2}}{r^{3} \pi_{z, k i n}}+e \frac{\pi_{\theta, k i n}}{r^{2} \pi_{z, k i n}} \frac{\partial\left(r A_{\theta}^{*}\right)}{\partial r} .
\end{aligned}
$$

The remaining transversal equations are:

$$
\begin{aligned}
& \frac{d r}{d z}=\frac{\partial K_{f}}{\partial \pi_{r, c a n}}=\frac{\pi_{r, k i n}}{\pi_{z, k i n}}, \\
& \pi_{\theta, k i n}=\pi_{\theta, \text { can }}(0)-e r A_{\theta}^{*}(r, z), \\
& \frac{d \theta}{d z}=\frac{\partial K_{f}}{\partial \pi_{\theta}}=\frac{\pi_{\theta, k i n}}{r^{2} \pi_{z, k i n}} .
\end{aligned}
$$

It is seen that $\pi_{r, k i n}$ is needed in the set of equations and not $\pi_{r, \text { can }}$. Furthermore, the expression $d \pi_{r, k i n} / d z$ is much simpler than the expression $d \pi_{r, c a n} / d z$. With this set of equations particle motion calculations can be performed.
The equations of motion for calculations without static external solenoidal magnetic field are obtained by setting $A_{\theta}$ equal to zero. Calculations without electromagnetic field are obtained by setting $\varepsilon_{n}(z)$ equal to zero. In this case the differential equation for energy changes in $h(z)=1$ and the differential equation for the phase does not have any meaning anymore, because of the absence of an electromagnetic field.

\section{CALCULATIONS COMPARED TO SIMULATIONS}

In this section calculations based on the Hamiltonian equations of motion of the previous section are presented and compared to the results of the particle-tracking code General Particle Tracer (GPT) [3]. In this code, the same electric and magnetic field description is specified as is used in the Hamiltonian calculations, however this particle-tracking code uses an entirely different calculation method. Calculations of motion in an electromagnetic field with and without static external magnetic field have been applied to the periodic part of the Eindhoven $10 \mathrm{MeV}$ linac.

The energy and phase have been calculated for the $10 \mathrm{MeV}$ linac using eq. 7 (fig. 1). Because of the small dependence of the particle distance to the $z$-axis on the calculation of energy and phase, particles with a different initial position and divergence will have similar energy and phase evolution. Calculations are performed at four different injection phases, with an initial energy of $1 \mathrm{MeV}$. Energy calculations are compared to the results of GPT, showing good agreement between both methods. The particle phase is not provided by GPT, so a comparison of phases is not possible. However, because of the agreement in energy calculations, it is obvious that the Hamiltonian phase calculations must be consistent.

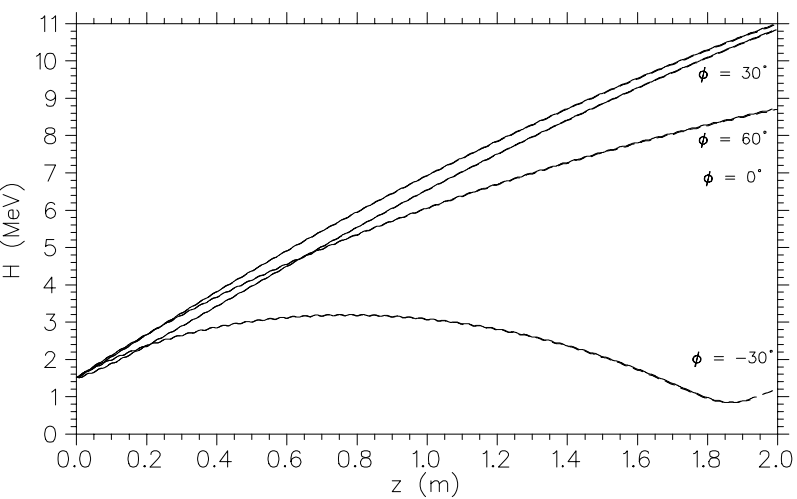

Figure 1: Particle energy $H$ as a function of longitudinal co-ordinate $z$ in the periodic part of the linac, at various injection phases. Hamilton calculations are presented by the dashed line, GPT simulations by the solid line. 
Fig. 2 depicts the calculations of the distance of a particle to the $z$-axis, $r$, and the divergence $r$ ' in the linac, using eqs. 9 and 10. Particles are injected at various phases at $1 \mathrm{MeV}$ parallel to the $z$-axis with an initial radius $r=3 \mathrm{~mm}$. It is shown that there is a good agreement between Hamiltonian calculations and GPT simulations.
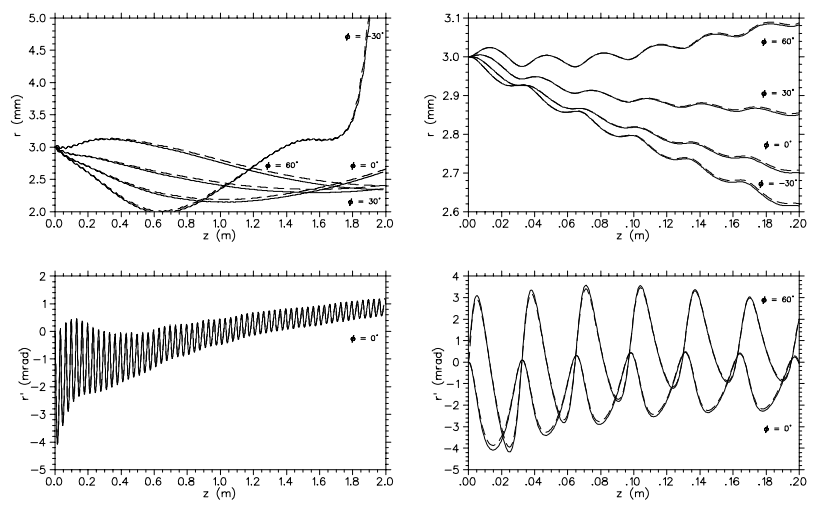

Figure 2: Particle distance to $z$-axis, $r$, and divergence, $r$, calculations as a function of $z$, for the entire periodic part of the linac (left figs.) and for the first six cells of the periodic part (right figs.) Hamilton calculations are presented by the dashed line, GPT simulations by the solid line.

Fig. 3 shows the azimuthal co-ordinate $\theta$ and its derivative $\theta^{\prime}$ as a function of $z$ in the linac for the same calculations as depicted in fig. 2. Again there is a good agreement between Hamiltonian calculations and GPT simulations.
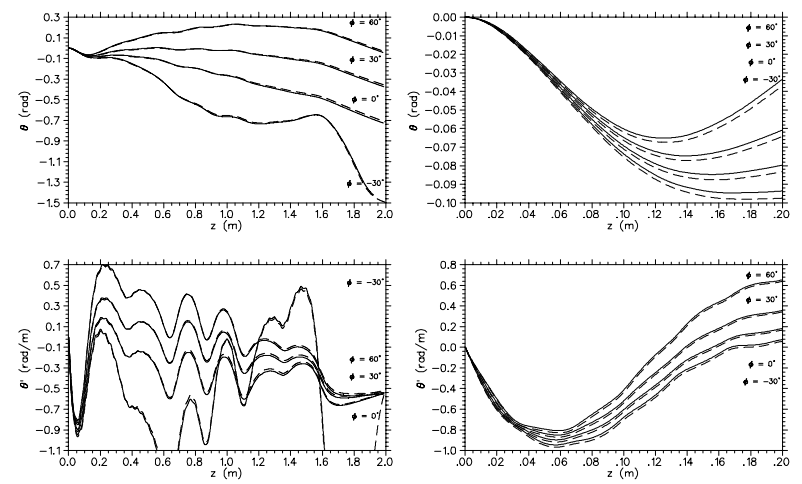

Figure 3: Particle azimuthal co-ordinate, $\theta$, and its derivative, $\theta$ ', calculations as a function of $z$, for the entire periodic part of the linac (left figs.) and for the first six cells of the periodic part (right figs.) Hamilton calculations are presented by the dashed line, GPT simulations by the solid line.

\section{CONCLUSIONS}

An analytic description of particle motion in linear accelerators has been developed, in an earlier paper restricted to higher energy standing wave structures, while in the present paper this has been extended to the case of low-energy travelling-wave linacs, in which solenoidal fields may be incorporated. The Hamiltonian calculations yield a remarkable agreement with results of particle-tracking codes but they are much faster performed than simulations by these codes.

\section{REFERENCES}

[1] A.F.J. Hammen, J.M. Corstens, J.I.M. Botman, H.L. Hagedoorn, W.H.C. Theuws, "Hamiltonian Calculations on Particle Motion in Linear Electron Accelerators", European Particle Accelerator Conference, Stockholm, Sweden (1998).

[2] R.W. de Leeuw, "The accelerator injection chain of the electron storage ring EUTERPE", Ph.D. Thesis, Eindhoven University of Technology, Eindhoven, The Netherlands (1996).

[3] General Particle Tracer, "User Manual", Pulsar Physics, Utrecht, The Netherlands (1997)

\section{APPENDIX}

Consider the following equations:

$\frac{d \pi_{r, k i n}}{d z}=\frac{d \pi_{r, c a n}}{d z}-e \frac{d A_{r}^{*}}{d z}$,

$\frac{d A_{r}^{*}}{d z}=\frac{\partial A_{r}^{*}}{\partial r} \frac{d r}{d z}+\frac{\partial A_{r}^{*}}{\partial z}+\frac{\partial A_{r}^{*}}{\partial t} \frac{1}{v_{z}}$,

$\frac{d r}{d z}=\frac{\pi_{r, k i n}}{\pi_{z, k i n}}$.

Thus, using eq. 8:

$$
\begin{aligned}
\frac{d \pi_{r, k i n}}{d z}= & e \frac{\partial A_{z}^{*}}{\partial r}-e \frac{\partial A_{r}^{*}}{\partial z}-e \frac{\partial A_{r}^{*}}{\partial t} \frac{1}{v_{z}}+\frac{\pi_{\theta, k i n}^{2}}{r^{3} \pi_{z, k i n}}+ \\
& e \frac{\pi_{\theta, k i n}}{r^{2} \pi_{z, k i n}} \frac{\partial\left(r A_{\theta}^{*}\right)}{\partial r} .
\end{aligned}
$$

Substitution of the vectorpotential given by eq. 1 yields:

$$
\begin{gathered}
\frac{d \pi_{r, k i n}}{d z}=\sum_{n} \varepsilon_{n} I_{1}\left(\alpha_{n} r\right)\left\{-\alpha_{n}+\frac{k_{n}^{2}}{\alpha_{n}}-\frac{\omega}{v_{z}} \frac{k_{n}}{\alpha_{n}}\right\} \sin \left(k_{n} z-\omega t\right)+ \\
\frac{\pi_{\theta, k i n}^{2}}{r^{3} \pi_{z, \text { kin }}}+e \frac{\pi_{\theta, \text { kin }}}{r^{2} \pi_{z, \text { kin }}} \frac{\partial\left(r A_{\theta}^{*}\right)}{\partial r} .
\end{gathered}
$$

in which the $z$-dependence of the amplitude in $A_{r}$ has been ignored. After some mathematical manipulation and replacement of the argument $\left(k_{n} z-\omega t\right)$ by $(2 \pi n z / d+k \zeta)$ the following expression holds:

$$
\begin{gathered}
\frac{d \pi_{r, k i n}}{d z}=\sum_{n} \varepsilon_{n} \frac{I_{1}\left(\alpha_{n} r\right)}{\alpha_{n}} k\left(k-k_{n} \frac{h}{\pi_{z, k i n}}\right) \sin \left(\frac{2 \pi n}{d} z+k \zeta\right)+ \\
\frac{\pi_{\theta, k i n}^{2}}{r^{3} \pi_{z, k i n}}+e \frac{\pi_{\theta, k i n}}{r^{2} \pi_{z, k i n}} \frac{\partial\left(r A_{\theta}^{*}\right)}{\partial r} .
\end{gathered}
$$

